\title{
Some Aspects Concerning the "Memorization Effect" in Complex Fluid
}

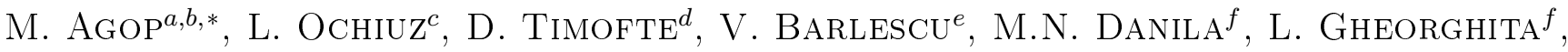 \\ V.P. PAUn ${ }^{g}$, L.G. SOlOVASTRU ${ }^{h}$ AND C. POPA ${ }^{i}$ \\ ${ }^{a}$ Lasers, Atoms and Molecules Physics Laboratory, University of Science and Technology, \\ Villeneuve d'Ascq 59655, Lille, France \\ ${ }^{b}$ Department of Physics, "Gh. Asachi" Technical University, D. Mangeron str. 67, Iaşi 700050, Romania \\ ${ }^{c}$ Faculty of Pharmacy, Department of Pharmaceutical Technology, University of Medicine and Pharmacy, \\ "Gr. T. Popa" University, Independentei 16, Iaşi 700115, Romania \\ ${ }^{d}$ Surgery Department, Hospital "Sf. Spiridon", University of Medicine and Pharmacy, \\ "Gr. T. Popa" University, Independentei 16, Iaşi 700115, Romania \\ ${ }^{e}$ Department of Computer Science and Engineering, "Gh. Asachi" Technical University, \\ D. Mangeron str. 27, Iaşi 700050, Romania \\ ${ }^{f}$ Faculty of Physics, Alexandru Ioan Cuza University of Iasi, Bd-ul Carol I no. 11, Iasi 700506, Romania \\ ${ }^{g}$ Department of Physics I, Faculty of Applied Science, Politehnica University of Bucharest, \\ 313 Splaiul Independentei Str., 060042, Bucharest, Romania \\ ${ }^{h}$ Department of Dermatology, University of Medicine and Pharmacy "Gr. T. Popa" University, \\ Independentei 16, Iaşi 700115, Romania \\ ${ }^{i}$ Dentistry Department, University of Medicine and Pharmacy "Gr. T. Popa" University, \\ Independentei 16, Iasi 700115, Romania
}

(Received February 4, 2014)

In the frame of a non-standard scale relativity model, the specific momentum, states density and internal energy conservations laws are obtained. The chaoticity, either through turbulence in the fractal hydrodynamics approach, or through stochasticization in the Schrödinger type approach, is generated only by the non-differentiability of the movement trajectories of the complex fluid entities. Using the conservation laws mentioned above, by numerical simulations, hysteretic type effects (dynamics of hysteretic cycles) occur.

DOI: $10.12693 /$ APhysPolA.126.663

PACS: 05.45.Df, 47.53.+n, 03.65.-w

\section{Introduction}

Fluids with non-linear viscous behaviors, as well as viscoelastic materials are complex fluids. A great variety of materials are categorized as complex fluids: colloidal fluids, polymers (elastomers, thermoplastics and composites), biological fluids (DNA, proteins, cells, dispersions of biopolymers and cells, human blood), foams, emulsions, gels, suspensions, micelar and liquid-crystal phases, molten materials. In this case the fluids do not obey to the hydrodynamic laws [1-3].

The dynamics of the complex fluid entities represent an interesting topic. Friction causes loss of energy. This phenomenon can be explained through a nonlinear function depending not only on normal forces but also on velocity. The main element in such a system is flow restriction caused by the accumulation of component entities. At low temperatures and high densities, the complex fluid

${ }^{*}$ corresponding author; e-mail: m.agop@yahoo.com entities rearranges causing irregular intervals of solid-like behavior followed by stress relaxation. Any small displacement of a single particle as well as any infinitesimal stress increase can cause transition from a stable state to a fluid-like behavior. Consequently, this type of dynamics becomes highly nonlinear in the surrounding nature [4].

Correspondingly, theoretical models of dynamics in complex fluids can become sophisticated and ambiguous [1-3]. However, such situation can be standardized if we consider that complexities in interaction processes impose various time resolution scales while the evolution pattern leads to different freedom degrees [5-8].

To develop a theoretical model we have to admit that complex fluids with chaotic behaviour can achieve self-similarity (space-time structures can appear) associated with strong fluctuations at all possible space-time scales [5-8]. Then, for time scales that prove to be larger if compared with the inverse of the highest Lyapunov exponent, the deterministic trajectories are replaced by a collection of potential routes. In its turn, the concept of "definite positions" is replaced by that of probability density [9-12]. A most impressive example in this respect refers to collision processes in complex fluid. Here, the 
dynamics of the entities can be described through the non-differentiable curves [9-11].

Since non-differentiability appears as a universal property of complex fluids, it is necessary to create a non-differentiable physics of complex fluids. Under such circumstances, if we consider that the complexity of interactions in the dynamics of complex fluids is replaced by non-differentiability, it is no longer necessary to use the whole classical "arsenal" of quantities from standard physics (differentiable physics) [1-3].

This topic was developed in $[13,14]$ using both the scale relativity theory (SRT) $[10,11]$ and non-standard scale relativity theory (NSRT) [15-29]. According to SRT or NSRT, the dynamics of complex fluids entities takes place on continuous but non-differentiable curves (fractal curves), so that all physical phenomena involved depend not only on space-time coordinates but also on space-time scale resolution. That is why physical quantities describing the dynamics of complex fluids can be considered as fractal functions $[10,11]$. Moreover, according to geodesics in a non-differentiable (fractal) space, the complex fluid entities may be reduced to and identified with their own trajectories (i.e. their geodesics) so that the complex fluids should behave as a special "fluid" lacking interactions - fractal fluid [10, 11].

In the present paper various theoretical aspects of complex fluid dynamics (patterns generation, hysteretic behaviours, etc.) were analyzed using the NSRT.

\section{Motion equation via non-differentiability}

We can simplify the dynamics of a complex fluid supposing that the complex fluid entities move on continuous but non-differentiable curves, i.e. fractal curves (for example, the Peano curve, the Koch curve or the Weierstrass curve [9-11]).

Once accepted such a hypothesis, the dynamics of the complex fluid entities are given by the fractal operator $\hat{\mathrm{d}} / \mathrm{d} t[15-29]$ :

$$
\frac{\hat{\mathrm{d}}}{\mathrm{d} t}=\frac{\partial}{\partial t}+\hat{\boldsymbol{V}} \cdot \nabla-\mathrm{i} \frac{\lambda^{2}}{\tau}\left(\frac{\mathrm{d} t}{\tau}\right)^{\left(\frac{2}{D_{\mathrm{F}}}\right)-1} \Delta,
$$

where

$$
\hat{\boldsymbol{V}}=\boldsymbol{V}_{\mathrm{D}}-\mathrm{i} \boldsymbol{V}_{\mathrm{F}}^{\prime}
$$

is the complex velocity, $\boldsymbol{V}_{\mathrm{D}}$ is the differentiable and resolution scale independent velocity, $\boldsymbol{V}_{\mathrm{F}}$ is the non-differentiable and resolution scale dependent velocity, $\hat{\boldsymbol{V}} \cdot \nabla$ is the convective term,

$$
\begin{aligned}
& \left(\frac{\lambda^{2}}{\tau}\right)\left(\frac{\mathrm{d} t}{\tau}\right)^{\left(\frac{2}{D_{\mathrm{F}}}\right)-1} \Delta=\left(\frac{\lambda^{2}}{\tau}\right)\left(\frac{\mathrm{d} t}{\tau}\right)^{\left(\frac{2}{D_{\mathrm{F}}}\right)-1} \\
& \times\left[\left(\frac{\partial^{2}}{\partial x^{2}}\right)+\left(\frac{\partial^{2}}{\partial y^{2}}\right)+\left(\frac{\partial^{2}}{\partial z^{2}}\right)\right]
\end{aligned}
$$

is the dissipative term, $D_{\mathrm{F}}$ is the fractal dimension of the movement curve, $\lambda$ is the space scale, $\tau$ is the time scale and $\lambda^{2} / \tau$ is a specific coefficient associated to the fractal-non-fractal transition. For $D_{\mathrm{F}}$ any definition can be used (the Hausdorff-Besikovici fractal dimension, the Kolmogorov fractal dimension, etc. [9-11]), but once accepted such a definition for $D_{\mathrm{F}}$, it has to be constant over the entire analysis of the complex fluid dynamics. In a particular case, for motions on the Peano curves, $D_{\mathrm{F}}=2$ [9-11] of the complex fluid entities, the fractal operator (1) reduces to Nottale's operator $(\hat{\mathrm{d}} / \mathrm{d} t)_{N}$ :

$$
\left(\frac{\hat{\mathrm{d}}}{\mathrm{d} t}\right)_{N}=\frac{\partial}{\partial t}+\hat{\boldsymbol{V}} \cdot \nabla-\mathrm{i} D_{N} \Delta
$$

where $D_{N}=\lambda^{2} / \tau$ is the Nottale coefficient associated to the fractal-non-fractal transition.

Applying the fractal operator (1) to the complex speed (2) and accepting the principle of scale covariance [10,11] in the form

$$
\frac{\hat{\mathrm{d}} \hat{\boldsymbol{V}}}{\mathrm{d} t}=0,
$$

we obtain the motion equation

$$
\begin{aligned}
& \frac{\hat{\mathrm{d}} \hat{\boldsymbol{V}}}{\mathrm{d} t}=\frac{\partial \hat{\boldsymbol{V}}}{\partial t}+(\hat{\boldsymbol{V}} \cdot \nabla) \hat{\boldsymbol{V}}-\mathrm{i} \frac{\lambda^{2}}{\tau}\left(\frac{\mathrm{d} t}{\tau}\right)^{\left(\frac{2}{D_{\mathrm{F}}}\right)-1} \\
& \quad \times \Delta \hat{V}=0 .
\end{aligned}
$$

It means that at any point of a fractal path, the local acceleration term, $\partial_{t} \hat{\boldsymbol{V}}$, the non-linear (convective) term, $(\hat{\boldsymbol{V}} \cdot \nabla) \hat{\boldsymbol{V}}$, and the dissipative term, $\left(\lambda^{2} / \tau\right)(\mathrm{d} t / \tau)^{\left(2 / D_{\mathrm{F}}\right)-1} \Delta \hat{V}$, make their balance. Therefore, the complex fluid is assimilated to a "rheological" fluid, whose dynamics are described by the complex velocities field, $\hat{\boldsymbol{V}}$, complex acceleration field, $\partial_{t} \hat{\boldsymbol{V}}$, etc. and by the imaginary viscosity type coefficient, $\mathrm{i}\left(\lambda^{2} / \tau\right)(\mathrm{d} t / \tau)^{\left(2 / D_{\mathrm{F}}\right)-1}$. The "rheology" of the fluid can provide hysteretic properties to the complex fluid (the complex fluid has a hysteresis cycle, memory, etc. [28]).

\section{Chaoticity via non-differentiability}

For irrotational motions of the complex fluid entities

$$
\nabla \times \hat{\boldsymbol{V}}=0, \quad \nabla \times V_{\mathrm{D}}=0, \quad \nabla \times V_{\mathrm{F}}=0,
$$
we can choose $\hat{\boldsymbol{V}}$ of the form

$$
\hat{\boldsymbol{V}}=-\mathrm{i} \frac{\lambda^{2}}{\tau}\left(\frac{\mathrm{d} t}{\tau}\right)^{\left(\frac{2}{D_{\mathrm{F}}}\right)-1} \nabla \ln \psi
$$

where $\varphi \equiv \ln \psi$ is the velocity scalar potential. By substituting (7) in (5) and using the method described in [28], it results that

$$
\begin{aligned}
& \frac{\mathrm{d} \hat{\boldsymbol{V}}}{\mathrm{d} t}=-\mathrm{i} \frac{\lambda^{2}}{\tau}\left(\frac{\mathrm{d} t}{\tau}\right)^{\left(\frac{2}{D_{\mathrm{F}}}\right)-1} \\
& \quad \times \nabla\left[\frac{\partial \ln \psi}{\partial t}-\mathrm{i} \frac{\lambda^{2}}{\tau}\left(\frac{\mathrm{d} t}{\tau}\right)^{\left(\frac{2}{D_{\mathrm{F}}}\right)-1} \frac{\nabla \psi}{\psi}\right]=0 .
\end{aligned}
$$

This equation can be integrated in a universal way and yields

$$
\frac{\lambda^{4}}{\tau^{2}}\left(\frac{\mathrm{d} t}{\tau}\right)^{\left(\frac{4}{D_{\mathrm{F}}}\right)-2} \Delta \psi+\mathrm{i} \frac{\lambda^{2}}{\tau}\left(\frac{\mathrm{d} t}{\tau}\right)^{\left(\frac{2}{D_{\mathrm{F}}}\right)-1} \frac{\partial \psi}{\partial t}=0,
$$


by a suitable choice of the phase of $\psi$. For motions of the complex fluid entities on the Peano curves, $D_{\mathrm{F}}=2$ [9] at the Compton scale $\lambda^{2} / \tau=\hbar / 2 m_{0}$ [10, 11], with $\hbar$ the reduced Plank constant and $m_{0}$ the rest mass of the complex fluid entities, the relation (9) becomes the standard Schrödinger equation

$$
\frac{\hbar^{2}}{2 m_{0}} \Delta \psi+\mathrm{i} \hbar \frac{\partial \psi}{\partial t}=0
$$

If $\psi=\sqrt{\rho} \mathrm{e}^{\mathrm{i} S}$, with $\sqrt{\rho}$ the amplitude and $S$ the phase of $\psi$, the complex velocity field ( 7 ) takes the explicit form

$$
\begin{aligned}
& \hat{\boldsymbol{V}}=\frac{\lambda^{2}}{\tau}\left(\frac{\mathrm{d} t}{\tau}\right)^{\left(\frac{2}{D_{\mathrm{F}}}\right)-1} \nabla S-\mathrm{i} \frac{\lambda^{2}}{2 \tau}\left(\frac{\mathrm{d} t}{\tau}\right)^{\left(\frac{2}{D_{\mathrm{F}}}\right)-1} \nabla \ln \rho \\
& \boldsymbol{V}_{\mathrm{D}}=\frac{\lambda^{2}}{\tau}\left(\frac{\mathrm{d} t}{\tau}\right)^{\left(\frac{2}{D_{\mathrm{F}}}\right)-1} \nabla S \\
& \boldsymbol{V}_{\mathrm{F}}=\frac{\lambda^{2}}{2 \tau}\left(\frac{\mathrm{d} t}{\tau}\right)^{\left(\frac{2}{D_{\mathrm{F}}}\right)-1} \nabla \ln \rho .
\end{aligned}
$$

By substituting (10)-(12) in (5) and separating the real and the imaginary parts, up to an arbitrary phase factor which may be set at zero by a suitable choice of the phase of $\psi$, we obtain

$$
\begin{aligned}
& \frac{\partial \boldsymbol{V}_{\mathrm{D}}}{\partial t}+\left(\boldsymbol{V}_{\mathrm{D}} \cdot \nabla\right) \boldsymbol{V}_{\mathrm{D}}=-\nabla(Q), \\
& \frac{\partial \rho}{\partial t}+\nabla \cdot\left(\rho \boldsymbol{V}_{\mathrm{D}}\right)=0,
\end{aligned}
$$

with $Q$ the specific fractal potential

$$
\begin{aligned}
Q & =-2 \frac{\lambda^{4}}{\tau^{2}}\left(\frac{\mathrm{d} t}{\tau}\right)^{\left(\frac{4}{D_{\mathrm{F}}}\right)-2} \frac{\Delta \sqrt{\rho}}{\sqrt{\rho}} \\
& =-\frac{\boldsymbol{V}_{\mathrm{F}}^{2}}{2}-\frac{\lambda^{2}}{\tau}\left(\frac{\mathrm{d} t}{\tau}\right)^{\left(\frac{2}{D_{\mathrm{F}}}\right)-1} \nabla \cdot \boldsymbol{V}_{\mathrm{F}} .
\end{aligned}
$$

Equation (13) represents the specific momentum conservation law, while Eq. (14) represents the states density conservation law. Through the fractal velocity, $\boldsymbol{V}_{\mathrm{F}}$, the specific fractal potential $Q$ is a measure of non-differentiability of the complex fluid entities trajectories, i.e. of their chaoticity. Equations (13)-(15) define the fractal hydrodynamics model (FHM). In such a context, the complex fluid is assimilated to a fractal fluid.

Now, certain conclusions are evident: (i) for motions of the complex fluid entities on the Peano curves at the Compton scale [10, 11], the FHM reduces to a quantum hydrodynamic model (QHM); (ii) the fractal velocity $\boldsymbol{V}_{\mathrm{F}}$ does not represent actual mechanical motion, but does contribute to the transfer of specific momentum and the concentration of energy. This may be seen clearly from the absence of $\boldsymbol{V}_{\mathrm{F}}$ from the states density conservation law, and from its role in the variational principle [10, 11]; (iii) any interpretation of $Q$ should take cognizance of the "self" or internal nature of the specific momentum transfer. While the energy is stored in the form of the mass motion and potential energy (as it is classically), some is available elsewhere and only the total is conserved. It is the conservation of energy and specific momentum that ensures reversibility and the existence of eigenstates, but denies a Brownian-motion [9] form of interaction with an external medium; (iv) for the Peano curves motions of the complex fluid entities at spatial scales higher than the dimension of the boundary layer and at temporal scales higher than the oscillation periods of the pulsating velocities which overlaps the average velocity of the complex fluid motions (for details see [1-3], the FHM reduces to the standard hydrodynamical model [30]). We remind that, in this approximation of motion and for a special complex fluid type, $\nabla Q=-\nabla \widehat{\sigma} / \rho$, with $\widehat{\sigma}$ is the internal stress tensor type. In particular case, $\widehat{\sigma}$ can be put in correspondence with the pressure $p ;(\mathrm{v})$ since the position vector of the complex fluid entity is assimilated with a stochastic process of Wiener type [9-11], $\psi$ is not only the scalar potential of a complex velocity (through $\varphi \equiv \ln \psi)$ in the frame of FHM, but also states density (through $|\psi|^{2}$ ) in the frame of a Schrödinger type model. It results the equivalence between the formalism of the FHM and the one of Schrödinger type. Moreover, the chaoticity, either through turbulence in the fractal hydrodynamics approach, or through stochasticization in the Schrödinger type approach, is generated only by the non-differentiability of the movement trajectories in a fractal space; (vi) in the standard model (Landau's scenario [30]) the Fourier spectrum is always discrete and cannot approximate a continuum spectrum that in case of a large number of frequencies will generate a unlimited number of spectral components as a result of their beats which appear thanks to the presence of nonlinearities in the complex fluid. Yet, considering standard model, the flow can never be truly chaotic because, in case of multiple periodic functions, correlations tend to be not null, but having an oscillating character. Therefore, Landau's scenario can describe transition towards chaotic behavior only in a complex fluid with an infinite number of degrees of freedom. In our case, when $\delta t / \tau \rightarrow 0$ for $D_{\mathrm{F}} \neq 2$ the physical quantities that describe the dynamics of the complex fluid are no longer defined. So, in this approximation, a simulation of a system with an infinite number of degrees of freedom is used. Moreover, dynamic states could be generated, characterized by windows of regular oscillations interrupted by chaotic bursts, the transition between the two states being spontaneous, unpredictable and independent of any of the control parameters variation (turbulence through intermittency).

\section{A numerical simulation}

In the following, using (13), (14) and (15) with $\nabla Q=$ $\nabla p / \rho$, we analyze the dynamics of the complex fluid in a plane symmetry. The presence of an external perturbation is specified by adequate initial and boundary conditions (e.g. spatio-temporal Gaussian). In this situation, let us introduce the normalized coordinates

$$
\omega t=\tau_{0}, \quad k x=\xi, \quad k y=\eta, \quad \frac{V_{x} k}{\omega}=V_{\xi},
$$




$$
\frac{V_{y} k}{\omega}=V_{\eta}, \quad \frac{\rho}{\rho_{0}}=N,
$$

where $\omega, k$, and $\rho_{0}$ are critical parameters of the complex fluid (for details see [1-3]).

Then, Eqs. (13) and (14) become

$$
\begin{aligned}
& \frac{\partial}{\partial \tau_{0}}\left(N V_{\xi}\right)+\frac{\partial}{\partial \xi}\left(N V_{\xi}^{2}\right)+\frac{\partial}{\partial \eta}\left(N V_{\xi} V_{\eta}\right)=-N^{-1} \frac{\partial N}{\partial \xi}, \\
& \frac{\partial}{\partial \tau_{0}}\left(N V_{\eta}\right)+\frac{\partial}{\partial \xi}\left(N V_{\xi} V_{\eta}\right)+\frac{\partial}{\partial \eta}\left(N V_{\eta}^{2}\right)=-N^{-1} \frac{\partial N}{\partial \eta}, \\
& \frac{\partial N}{\partial \tau_{0}}+\frac{\partial}{\partial \xi}\left(N V_{\xi}\right)+\frac{\partial}{\partial \eta}\left(N V_{\eta}\right)=0 .
\end{aligned}
$$

For the numerical integration we shall impose the initial conditions

$$
\begin{gathered}
V_{\xi}(0, \xi, \eta)=0, \quad V_{\eta}(0, \xi, \eta)=0, \quad N(0, \xi, \eta)=1 / 5, \\
1 \leq \xi \leq 2, \quad 0 \leq \eta \leq 1,
\end{gathered}
$$

as well as the boundary conditions

$$
\begin{aligned}
& V_{\xi}\left(\tau_{0}, 1, \eta\right)=V_{\xi}\left(\tau_{0}, 2, \eta\right)=0, \\
& V_{\eta}\left(\tau_{0}, 1, \eta\right)=V_{\eta}\left(\tau_{0}, 2, \eta\right)=0, \\
& V_{\xi}\left(\tau_{0}, \xi, 0\right)=V_{\xi}\left(\tau_{0}, \xi, 1\right)=0, \\
& V_{\eta}\left(\tau_{0}, \xi, 0\right)=V_{\eta}\left(\tau_{0}, \xi, 1\right)=0, \\
& N\left(\tau_{0}, 1, \eta\right)=N\left(\tau_{0}, 2, \eta\right)=1 / 5, \\
& N\left(\tau_{0}, \xi, 0\right)=\frac{1}{10} \exp \left(-\left(\frac{\tau_{0}-1 / 5}{1 / 5}\right)^{2}\right) \\
& \times \exp \left(-\left(\frac{\xi-3 / 2}{1 / 5}\right)^{2}\right),
\end{aligned}
$$$$
N\left(\tau_{0}, \xi, 1\right)=1 / 5 \text {. }
$$

By using the finite differences method [31], the system $(17 \mathrm{a}-\mathrm{c})$ with the initial conditions $(18 \mathrm{a}-\mathrm{e})$ and the boundary ones $(19 \mathrm{a}-\mathrm{g})$ was numerically resolved.

In Fig. 1a,c,e three-dimensional dependences of the normalized density $N$, normalized velocities, $V_{\xi}$ and $V_{\eta}$, on the normalized coordinates, $\xi$ and $\eta$ are given for the normalized time $\tau_{0}=0.65$. Also in Fig. $1 \mathrm{~b}, \mathrm{~d}$,f the two-dimensional contour of the normalized density $N$, normalized velocities, $V_{\xi}$ and $V_{\eta}$, are given for the same normalized time. The following results: (i) the two patterns generation; (ii) the symmetry of the normalized velocity, $V_{\xi}$, with respect of symmetry axis of the spatial-temporal Gaussian; (iii) vertices at the patterns periphery for the normalized velocity field, $V_{\eta}$.

In our opinion, the patterns generation is a consequence of the self-structuring in complex fluids $[1-3,32-34]$.

\section{Hysteretic type behaviours via non-differentiability}

Applying the fractal operator (1) to the internal energy per unit volume, $\varepsilon$, and adopting the principle of scale covariance [10, 11], we obtain the internal energy per unit volume conservation law
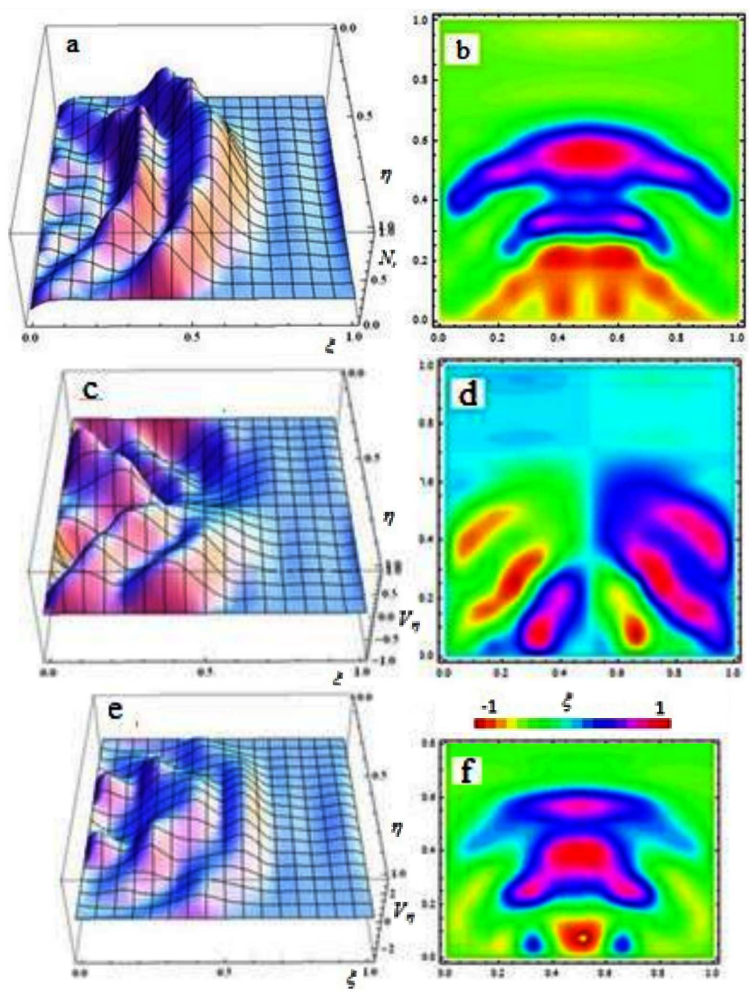

Fig. 1. (a)-(f) Three-dimensional dependences and two-dimensional contour of the normalized density $N$ $(\mathrm{a}, \mathrm{b})$, normalized velocity $V_{\xi}(\mathrm{c}, \mathrm{d})$ and normalized velocity $V_{\eta}(\mathrm{e}, \mathrm{f})$ on the normalized coordinates, $\xi$ and $\eta$ for the normalized time $\tau_{0}=0.65$.

$$
\begin{aligned}
& \frac{\hat{\mathrm{d}}(\rho \varepsilon)}{\mathrm{d} t}=\frac{\partial(\rho \varepsilon)}{\partial t}+(\hat{\boldsymbol{V}} \cdot \nabla)(\rho \varepsilon) \\
& -\mathrm{i} \frac{\lambda^{2}}{\tau}\left(\frac{\mathrm{d} t}{\tau}\right)^{\left(\frac{2}{D_{\mathrm{F}}}\right)-1} \Delta(\rho \varepsilon)=0 .
\end{aligned}
$$

For the types of movements mentioned in Sect. 4, separating the real part from the imaginary one in Eq. (20), we shall obtain

$$
\begin{aligned}
& \frac{\partial(\rho \varepsilon)}{\partial t}+\nabla\left(\rho \varepsilon \boldsymbol{V}_{\mathrm{D}}\right)=(\rho \varepsilon) \nabla \boldsymbol{V}_{\mathrm{D}}, \\
& \boldsymbol{V}_{\mathrm{F}} \cdot \nabla(\rho \varepsilon)=-\frac{\lambda^{2}}{\tau}\left(\frac{\mathrm{d} t}{\tau}\right)^{\left(\frac{2}{D_{\mathrm{F}}}\right)-1} \Delta(\rho \varepsilon) .
\end{aligned}
$$

One can notice that, although there is internal energy per unit volume transport at differentiable scale, a similar phenomenon (convection transport) at fractal scale occurs.

Let us reconsider Eqs. (13), (14) and (21) in a plane symmetry for $\nabla Q=\nabla p / \rho$ and let us assume that the variation of $p$ is induced by the variations of internal energy per unit volume and states density, $\nabla p=\nu \nabla(\rho \varepsilon)$, with $\nu=$ const.

Then, in dimensionless variables,

$$
\omega t=\tau, \quad k x=\xi, \quad k y=\eta,
$$




$$
\frac{V_{x} k}{\omega}=V_{\xi}, \quad \frac{V_{y} k}{\omega}=V_{\eta}, \frac{\rho}{\rho_{0}}=N, \quad \frac{\varepsilon}{\varepsilon_{0}}=\Theta,
$$

Eqs. (13), (14) and (21) become

$$
\begin{aligned}
& \frac{\partial}{\partial \tau}\left(N V_{\xi}\right)+\frac{\partial}{\partial \xi}\left(N V_{\xi}^{2}\right)+\frac{\partial}{\partial \eta}\left(N V_{\xi} V_{\eta}\right)=-\frac{\partial(N \Theta)}{\partial \xi} \\
& \frac{\partial}{\partial \tau}\left(N V_{\eta}\right)+\frac{\partial}{\partial \xi}\left(N V_{\xi} V_{\eta}\right)+\frac{\partial}{\partial \eta}\left(N V_{\eta}^{2}\right)=-\frac{\partial(N \Theta)}{\partial \eta} \\
& \frac{\partial N}{\partial \tau}+\frac{\partial}{\partial \xi}\left(N V_{\xi}\right)+\frac{\partial}{\partial \eta}\left(N V_{\eta}\right)=0 \\
& \frac{\partial(N \Theta)}{\partial \tau}+\frac{\partial}{\partial \xi}\left(N \Theta V_{\xi}\right)+\frac{\partial}{\partial \eta}\left(N \Theta V_{\eta}\right) \\
& =N \Theta\left(\frac{\partial V_{\xi}}{\partial \xi}+\frac{\partial V_{\eta}}{\partial \eta}\right)
\end{aligned}
$$

where the functional scaling relation, $\nu k^{2} / \omega^{2}=1$, was considered. In Eqs. (23f,g) and (24)-(27) $\rho_{0}$ and $\varepsilon_{0}$ correspond to equilibrium density and equilibrium internal energy per unit area of the complex fluid. For numerical integration, we consider the initial conditions,

$$
\begin{aligned}
& V_{\xi}(0, \xi, \eta)=0, \quad V_{\eta}(0, \xi, \eta)=0, \\
& N(0, \xi, \eta)=1 / 4, \quad \Theta(0, \xi, \eta)=1 / 4, \\
& \quad 0 \leq \xi \times \eta \leq 1 \times 1,
\end{aligned}
$$

and the boundary ones,

$$
\begin{aligned}
& V_{\xi}(\tau, 0, \eta)=0, \quad V_{\xi}(\tau, 1, \eta)=0, \\
& V_{\eta}(\tau, 0, \eta)=0, \quad V_{\eta}(\tau, 1, \eta)=0, \\
& N(\tau, 0, \eta)=1 / 4, \quad N(\tau, 1, \eta)=1 / 4, \\
& \Theta(\tau, 0, \eta)=1 / 4, \quad \Theta(\tau, 1, \eta)=1 / 4, \\
& V_{\xi}(\tau, \xi, 0)=0, \quad V_{\xi}(\tau, \xi, 1)=0, \\
& V_{\eta}(\tau, \xi, 0)=0, \quad V_{\eta}(\tau, \xi, 1)=0 \text {, } \\
& N(\tau, \xi, 0)=N_{0} \exp \left(-\frac{(\tau-1 / 4)^{2}}{(1 / 4)^{2}}\right) \\
& \times \exp \left(-\frac{(\xi-1 / 2)^{2}}{(1 / 4)^{2}}\right), \\
& N(\tau, \xi, 1)=1 / 4 \text {, } \\
& \Theta(\tau, \xi, 0)=\Theta_{0} \exp \left(-\frac{(\tau-1 / 4)^{2}}{(1 / 4)^{2}}\right) \\
& \times \exp \left(-\frac{(\xi-1 / 2)^{2}}{(1 / 4)^{2}}\right), \\
& \Theta(\tau, \xi, 1)=1 / 4 \text {. }
\end{aligned}
$$

In the boundary condition $(29 \mathrm{~m}, \mathrm{o})$ we assumed that the perturbation has a space-time Gaussian profile, $N_{0}$ is the maximum normalized states density and $\Theta_{0}$ is the maximum normalized internal energy per unit volume.

Equations (24)-(27) with the initial conditions (28a-e) and the boundary ones $(29 a-p)$ were numerically integrated via finite differences [31]. By means of numerical solutions in Fig. $2 \mathrm{a}-\mathrm{l}$ the three-dimensional dependences and two-dimensional contour of the normalized states density $N(\mathrm{a}, \mathrm{b})$, normalized internal energy per
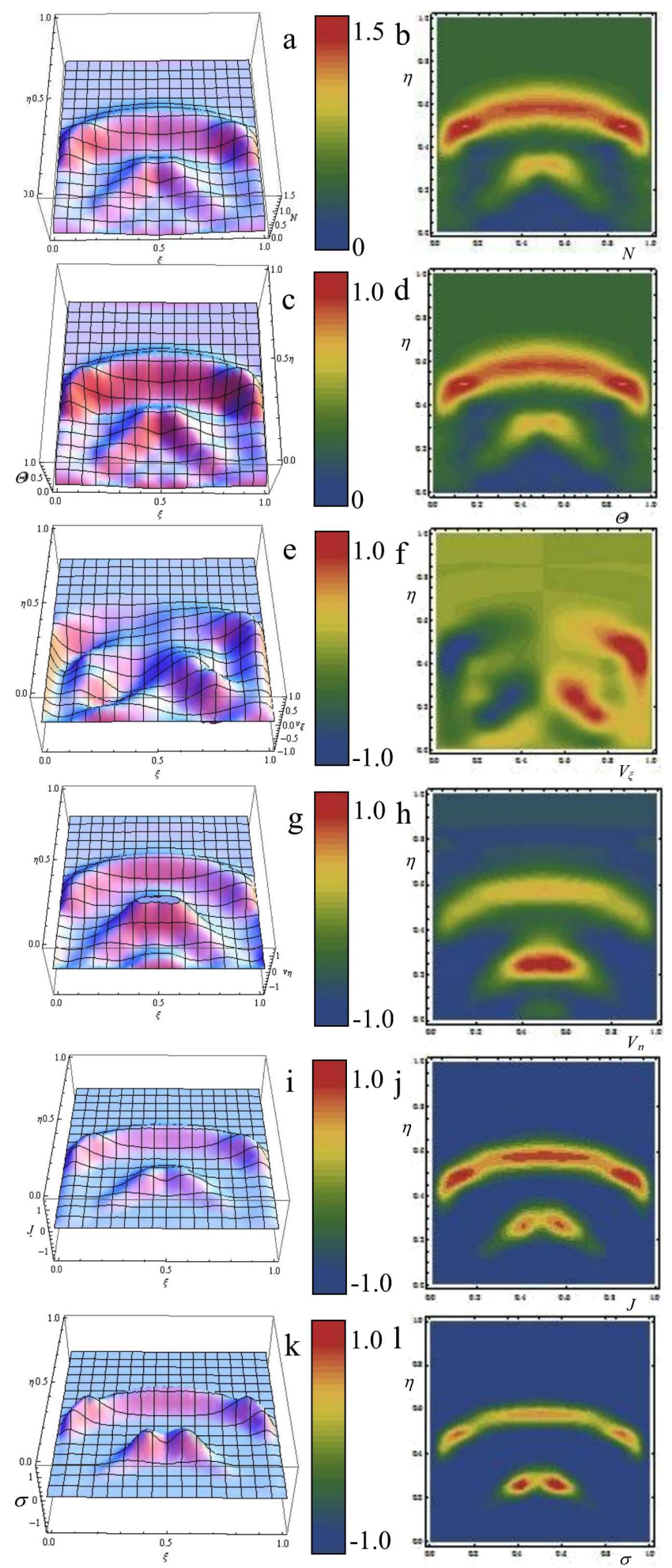

Fig. 2. (a)-(l) Three-dimensional dependences and two-dimensional contour of the normalized states density $N(\mathrm{a}, \mathrm{b})$, normalized internal energy per unit volume $\Theta(\mathrm{c}, \mathrm{d})$, normalized velocity $V_{\xi}(\mathrm{e}, \mathrm{f})$, normalized velocity $V_{\eta}(\mathrm{g}, \mathrm{h})$, normalized current density $J=N\left(V_{\xi}^{2}+\right.$ $\left.V_{\eta}^{2}\right)^{1 / 2}(\mathrm{i}, \mathrm{j})$, and diagonal component of the normalized internal stress tensor type $\sigma=N\left(V_{\xi}^{2}+V_{\eta}^{2}\right)(\mathrm{k}, \mathrm{l})$ on the normalized spatial coordinates $(\xi, \eta)$ at the normalized time $\tau=0.65$ for $N_{0}=1$ and $\Theta_{0}=1$. 
unit volume $\Theta(\mathrm{c}, \mathrm{d})$, normalized velocity $V_{\xi}(\mathrm{e}, \mathrm{f})$, normalized velocity $V_{\eta}(\mathrm{g}, \mathrm{h})$, normalized current density $J=N\left(V_{\xi}^{2}+V_{\eta}^{2}\right)^{1 / 2}(\mathrm{i}, \mathrm{j})$ and diagonal component of the normalized internal stress tensor type $\sigma=N\left(V_{\xi}^{2}+V_{\eta}^{2}\right)$ $(\mathrm{k}, \mathrm{l})$ on the normalized spatial coordinates $(\xi, \eta)$ at the normalized times $=0.65$ for $N_{0}=1$ and $\Theta_{0}=1$ are plotted. The following results are obtained:

(i) the generation of structures in complex fluid by means of solitons packet solutions $[35,36]$ ) (see the peaks from Fig. 2a, c, e, g, i, $\mathrm{k}$ and pronounced contours from Fig. 2b, d, f, h, j, l;

(ii) The normalized velocity $V_{\xi g} O$ which is normal to the "complex fluid streamline" is symmetric with respect to the symmetry axis of the space-time Gaussian, while vertices are induced at the periphery of the structures of the normalized velocity $V_{\eta}$ (which is along the "complex fluid streamline";

(iii) potential movement couplings at fractal scale as well as the potential one at differentiable scale are performed through the internal stress tensor type. As a result, the complex fluid entity acquires additional kinetic energy (induced by non-differentiability) that allows jumps from its own "stream line" to another;

(iv) eliminating the normalized time between the diagonal component of the normalized internal stress tensor type and normalized internal energy per unit volume for various given positions, one can obtain hysteresis type effects by numerical simulations. These effects are different for the $O \xi$ and $O \eta$ directions. For the same $\eta$, the dynamics of the hysteresis cycle are given in Fig. 3a-c. For the same $\xi$, such a tendency is more emphasized for small $\eta$ (Fig. 3d - hysteresis cycle), while for bigger $\eta$ it vanishes (Fig. 3f - absence of hysteresis cycle).

\section{Conclusions}

The main conclusions of the present paper are as follows:

i) assuming that the particle movements of a complex fluid occur on continuous but non-differentiable curves, the specific momentum, states density and internal energy conservation laws are obtained;

ii) for irrotational motion the chaoticity, either through turbulence in the fractal hydrodynamics approach, or through stochasticization in the Schrödinger type approach, is generated only by the non-differentiability of the movement's trajectories of the complex fluid entities;

iii) by numerical simulations using the FHM, the generation of structures in complex fluid by means of solitons packet solutions, the symmetry of the velocity field with respect of symmetry axis of a space-time Gaussian and vertices at the structures periphery of the velocity field are obtained;

iv) by numerical simulations using the FHM and internal energy per unit volume conservation law, one

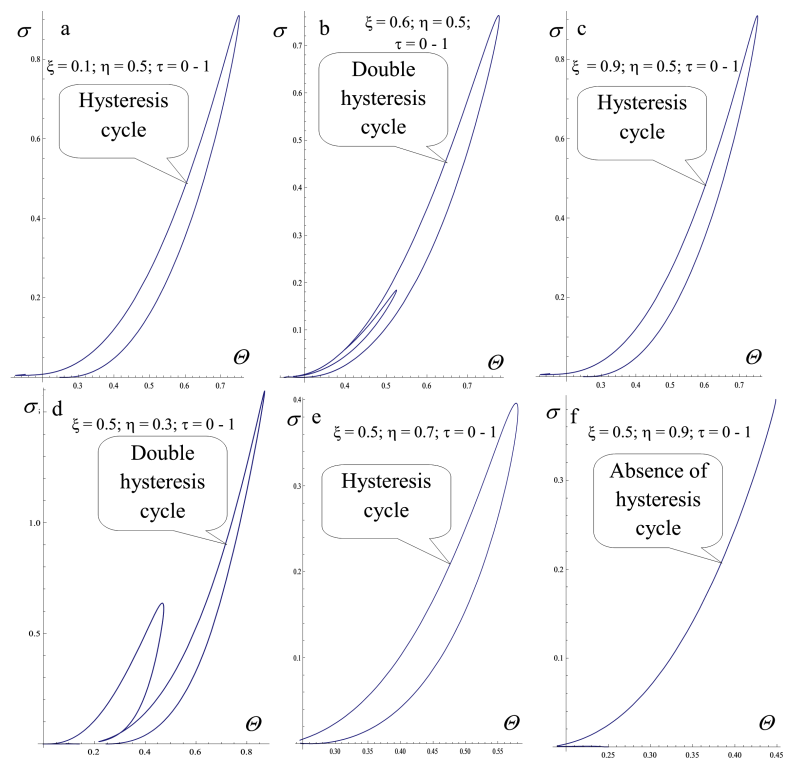

Fig. 3. (a)-(f) The dependences of the normalized internal stress tensor type $\sigma$ on the normalized internal energy per unit volume $\Theta$ for $\xi=0.1 ; \eta=0.5$; $\tau=0-1(\mathrm{a}), \xi=0.6 ; \eta=0.5 ; \tau=0-1(\mathrm{~b}), \xi=0.9$; $\eta=0.5 ; \tau=0-1$ (c), $\xi=0.5 ; \eta=0.3 ; \tau=0-1$ (d), $\xi=0.5 ; \eta=0.7 ; \tau=0-1(\mathrm{e}), \xi=0.5 ; \eta=0.9$ $\tau=0-1$ (f).

can obtain hysteresis type effects for various given positions (dynamics of hysteresis cycles).

\section{References}

[1] G. Luis, Complex Fluids, Springer, 1993, p. 415.

[2] T.Y. Hou, Multi-Scale Phenomena in Complex Fluids: Modeling, Analysis and Numerical Simulations, World Sci., Singapore 2009.

[3] O.D. Mitchell, B.G. Thomas, Mathematical Modeling for Complex Fluids and Flows, Springer, Berlin 2012.

[4] N. Keim, M. Garcia, P.E. Arratia, Phys. Fluids 24, 081703 (2012).

[5] C.H. Bennett, in: Complexity, Entropy, and the Physics of Information, Ed. W.H. Zurek, AddisonWesley, Reading, MA, 1990, p. 443.

[6] R. Badii, A. Politi, Complexity: Hierarchical Structure and Scaling in Physics, Cambridge Nonlinear Science Series, 6, Cambridge University Press, Cambridge 1997.

[7] G.W. Flake, The Computational Beauty of Nature, MIT Press, Cambridge, MA 1998.

[8] M. Mitchell, Complexity: A Guided Tour, Oxford University Press, Oxford 2009.

[9] B. Mandelbrot, The Fractal Geometry of Nature, W.H. Freeman, New York 1983.

[10] L. Nottale, Fractal Space-Time and Microphysics: Towards a Theory of Scale Relativity, World Sci., Singapore 1993. 
[11] L. Nottale, Scale Relativity and Fractal Space-Time - A New Approach to Unifying Relativity and Quantum Mechanics, Imperial College Press, London 2011.

[12] M.S. El Naschie, O.E. Rössler, I. Prigogine, Quantum Mechanics, Diffusion and Chaotic Fractals, Elsevier, Oxford 1995.

[13] V. Nedeff, E. Moşneguţu, M. Panainte, M. Ristea, G. Lazăr, D. Scurtu, B. Ciobanu, A. Timofte, S. Toma, M. Agop, Powder Technol. 221, 312 (2012).

[14] V. Nedeff, C. Bejenariu, G. Lazar, M. Agop, Powder Technol. 235, 685 (2013).

[15] M. Agop, L. Chicos, M. Gîrţu, Acta Phys. Pol. A 112, 3 (2007).

[16] M. Agop, P. Nica, A. Harabagiu, Acta Phys. Pol. A 113, 1571 (2008).

[17] A. Timofte, I. Casian Botez, D. Scurtu, M. Agop, Acta Phys. Pol. A 119, 304 (2011).

[18] M. Colotin, G.O. Pompilian, P. Nica, S. Gurlui, V. Paun, M. Agop, Acta Phys. Pol. A 116, 157 (2009).

[19] M. Agop, P. Nica, O. Niculescu, D.G. Dumitru, J. Phys. Soc. Japan 81, 064502 (2012).

[20] M. Agop, N. Forna, I. Casian-Botez, J. Comp. Theor. Nanostruct. 5, 483 (2008).

[21] M. Agop, C. Murgulet, Chaos Solitons Fractals 32, 1231 (2007)

[22] M. Agop, P. Nica, M. Girtu, Gen. Rel. Gravit. 40, 35 (2008)

[23] M. Agop, V. Paun, A. Harabagiu, Chaos Solitons Fractals 37, 1269 (2008).
[24] I. Casian-Botez, M. Agop, P. Nica, V. Paun, G.V. Munceleanu, J. Comp. Theor. Nanostruct. 7, 2271 (2010)

[25] C. Ciubotariu, M. Agop, Gen. Rel. Gravit. 28, 405 (1996).

[26] I. Gottlieb, M. Agop, M. Jarcau, Chaos Solitons Fractals 19, 705 (2004).

[27] S. Gurlui, M. Agop, M. Strat, S. Bacaita, Phys. Plasmas 13, 705 (2006)

[28] G.V. Munceleanu, V.P. Paun, I. Casian-Botez, M. Agop, Int. J. Bifurc. Chaos 21, 603 (2011).

[29] S. Bacaita, C. Uritu, M. Popa, A. Uliniuc, C. Peptu, M. Agop, Smart Mater. Res. 2012, 26460948 (2012).

[30] L. Landau, E.M. Lifshitz, Fluid Mechanics, 2nd ed. Butterworth-Heinemann, Oxford 1987.

[31] O.C. Zinkiewicz, R.L. Taylor, J.Z. Zhu, The Finite Element Method: Its Basis and Fundamentals, Butterworth-Heinemann, Oxford 2005.

[32] P. Nica, M. Agop, S. Gurlui, C. Bejinariu, C. Focsa, Jpn. J. Appl. Phys. 51, 106102 (2012).

[33] P. Nica, P. Vizureanu, M. Agop, S. Gurlui, C. Focsa, N. Forna, P.D. Ioannou, Z. Borsos, Jpn. J. Appl. Phys. 48, 066001 (2009).

[34] S. Gurlui, M. Agop, P. Nica, M. Ziskind, C. Focşa, Phys. Rev. E 78, 026405 (2008).

[35] C.P. Cristescu, Nonlinear Dynamics and Chaos: Theoretical Fundaments and Applications, Romanian Acad. Pub. House, Bucuresti 2008.

[36] E.A. Jackson, Perspectives in Nonlinear Dynamics, Cambridge Univ. Press, Cambridge 1991. 